\title{
RÓTULOS DE ALIMENTOS INFANTIS: ALGUNS ASPECTOS DAS PRÁTICAS DE MARKETING NO BRASIL'
}

\author{
INFANT FOOD LABELS: SOME ASPECTS OF MARKETING PRACTICES IN BRAZIL
}

\author{
Tereza Setsuko TOMA² \\ Marina Ferreira REA ${ }^{2}$
}

\begin{abstract}
RESUMO
Foi realizado um estudo com o objetivo da avaliar como as indústrias tem se adaptado à Norma Brasileira de Comercialização de Alimentos para Lactentes no que diz respeito à rotulagem e embalagem de seus produtos. Em 32 cidades de 13 estados brasileiros no periodo de 1995 a 1996, cerca de 125 produtos compreendendo mamadeiras, bicos, chupetas, alimentos infantis, fórmulas e leites fluidos foram analisados. Observou-se alguma tentativa de adequação à Norma, comparado a avaliações similares realizadas anteriormente. No entanto, diversos produtos permaneceram com rótulos em desacordo com as regras em todos os tipos estudados, destacando-se o caso de mamadeiras e chupetas. Nos produtos onde se exige "advertência", as vezes estas são observadas, mas precisam ser revistas devido ao não destaque que elas recebem. Monitoramentos como este devem ser periódicos para que os órgãos fiscalizadores da vigilância Sanitária recebam subsídios para um trabalho continuado e necessário de proteção contra o marketing inapropriado de substitutos do leite materno.
\end{abstract}

Termos de Indexação: alimentos infantis, rotulagem de alimentos, substitutos do leite humano, aleitamento materno.

\begin{abstract}
A monitoring study of the Brazilian Code of Marketing of Breast Milk Substitutes was carried out in 1995-1996 with the objective of verifying how companies are in compliance with the Code in the question of labeling and packaging their products. In 32 cities of 13 states, 125 products including feeding bottles, teats, pacifiers, infant food, formula and fluid milk were analyzed. A certain compliance was observed, in comparison to previous evaluations, done with the same methodological procedures. However, several products have not adequate labels, particularly feeding bottles and pacifiers. Rules related to "warning" need a review, because when they are accomplished, very often they are not
\end{abstract}

(1) Parte do Projeto Internacional: Monitoramento do Código de Comercialização de Alimentos para Lactentes, financiado pelo UNICEF.

(2) Pesquisadoras científicas, Instituto de Saúde, Secretaria de Estado da Saúde de São Paulo e Membros da Rede lnternational Baby Food Action Network (IBFAN). Correspondência para TSToma, Núcleo de Investigação em Saúde da Mulher e da Criança, Instituto de Saúde, Rua Santo Antônio, 590, $2^{\circ}$ andar, 01314-000, São Paulo, SP. 
easily seen. Routine monitoring procedures are needed to assist the competent authorities to protect breast feeding against the inappropriate marketing of commercial breast-feeding substitutes.

Key words: Infant food, food labeling, milk substitutes, breast feeding.

\section{INTRODUÇÃO}

As práticas de marketing de alimentos infantis têm assumido, nos últimos anos, diferentes aspectos. $\mathrm{Se}$, ao seremindustrializados no final do século passado, respondeu-se à crescente absorção da mão-de-obra feminina e conseqüente necessidade de facilitar a forma de alimentar a criança, com o correr do tempo observa-se que seus produtores foram buscando intensamente novos mercados para manter suas vendas e lucros (REA,1990). Assim, técnicas cada vez mais sofisticadas de marketing foram surgindo e estão em constante inovação.

A utilização de estratégias de marketing tais como rótulo ou embalagem atraentes, fornecimento de vantagens aos gerentes dos pontos de venda, e publicidade em quaisquer meios de comunicação de massa, exigem, atualmente que a vigilância sanitária nutricional e alimentar, componente importante da Saúde Pública, assim como as associações de consumidores, estejam atentas aos alimentos industrializados infantis e às mensagens promocionais que os acompanham.

As primeiras advertências encontradas na literatura contra a rotulagem de alimentos infantis datam do início do século, quando Coutts, em 1911, na Grã-Bretanha, propôs a colocação do aviso "IMPRÓPRIO PARA CRIANÇAS" nos rótulos de leite condensado desnatado (PALMER,1988). Isso ocorreu cerca de 40 anos depois que os doutores Platt e Daly, publicaram um artigo no periódico Lancet de 1872 , advertindo quanto ao desenvolvimento não satisfatório das crianças alimentadas com esse leite pelo seu baixo teor de gordura (DALY,1872; PLATT, 1872). Este fato antecipa a lentidão com que se incorporam conhecimentos científicos às práticas de consumo e vendas, e antecede de longa data a adoção, pela Assembléia Mundial de Saúde da Organização Mundial da Saúde (OMS) em 1981, do Código Internacional de Comercialização de Substitutos do Leite Materno (ORGANIZAÇÃO..., 1981). É interessante notar que esse Código constitui o único instrumento de recomendação do controle das práticas de marketing de produtos que interferem na saúde adotado pela OMS em toda a sua história. Em que pesem discussões e resoluções anuais aprovadas quanto a temas como o marketing do tabaco e de medicamentos, foi a questão dos alimentos infantis e sua interferência com o "produto" natural "leite humano" que conseguiu arregimentar adeptos para adoção de um Código de ética.

O período que antecede a adoção do Código encontra-se amplamente documentado por diversos autores (JELLIFFE, 1972; GREINER, 1975 CHETLEY, 1986; DOBBING, 1988;PALMER, 1988) inclusive nacionais (KUCINSKI, 1977; GOLDENBERG, 1988; REA, 1990) os quais de maneira geral, identificam a luta das indústrias pela comercialização de substitutos do leite materno com técnicas de marketing nem sempre éticas, como um dos principais fatores que levaram à diminuição das práticas de amamentação em nossos dias.

Por outro lado, pouquíssimos países - 24 até 1996, dentre os quais o Brasil - adotaram na totalidade as recomendações do Código consideradas pela OMS como "requerimentos mínimos"(ORGANIZAÇÃO..., 1981; INTERNATIONAL...,1996).

Em dezembro de 1988, o Brasil aprovou oficialmente o Código denominado Norma Brasileira para Comercialização de Alimentos para Lactentes. Após alguns anos de dificuldades na sua aplicação, esta Norma foi revisada e re-editada como Resolução do Conselho Nacional de Saúde 31/92, de 12 de outubro de 1992 (INSTITUTO...,1993). O objetivo desta Norma, assim como do Código Internacional é:

“...contribuir para a adequada nutrição dos lactentes e para defendê- los dos riscos associados à não amamentação ou ao desmame precoce, protegendo e incentivando a amamentação, mediante a regulamentação da promoção comercial e uso apropriado dos alimentos que são colocados à venda como substitutos ou complementos do leite materno."

Com o referencial teórico acima, diversos estudos de campo em várias partes do mundo, inclusive 
no Brasil, têm sido feitos para documentar e analisar o que tem havido com as práticas de marketing de alimentos infantis na vigência de "Códigos" ou regras claras de comercialização.

Este trabalho visa analisar os rótulos e embalagens de produtos comercializados como alimentos infantis, mamadeiras, bicos e chupetas, tendo como referência a Norma Brasileira para Comercialização de Alimentos para Lactentes (INSTITUTO..., 1993).

\section{MATERIAL E MÉTODOS}

Em 32 cidades brasileiras de 13 estados, desde o Pará até o Rio Grande do Sul, do final de 1995 ao início de 1996, pessoas foram treinadas sobre o conteúdo da Norma e capacitadas para a realização de um levantamento dos produtos industriais usados na alimentação infantil e na substituição do ato de sugar (mamadeiras, bicos e chupetas). Cento e vinte cinco produtos diferentes foram listados e analisados, a acordo com um formulário para identificação e classificação, tomando como referência os artigos pertinentes da Norma (TOMA,1996). Dos 29 artigos da Norma, 7 se aplicam a rotulagem. Estes serão apresentados com os comentários e análise.

\section{RESULTADOS E DISCUSSÃO}

Os produtos estudados no sentido de verificar cumpriam a Norma quanto à rotulagem foram classificados nos seguintes grupos:

1. Dezesseis tipos de leite infantil modificado ou especial.

2. Dezessete tipos de leite em pó, pasteurizado ou esterelizado.

3. Vinte e seis tipos de outros alimentos complementares.

4. Vinte e seis tipos de mamadeiras, doze tipos de bicos, vinte e quatro tipos de chupetas, quatro tipos de copos ou conjuntos para treinamento.

Nos pontos de venda, o rótulo chama a atenção do consumidor para o produto, podendo ser fator decisivo para influenciar sua compra. $\mathrm{O}$ nome do produto, as cores da embalagem, as imagens estampadas e as informações oferecidas fazem parte da idealização do rótulo, com o objetivo de atrair o cliente. O rótulo costuma ser também um veículo de informação ao cliente, embora muito usado como forma de promover o produto.

Dentro da lei e a partir das diretrizes da Resolução 31/92 (INSTITUTO..., 1993), os rótulos dos quatro grupos de produtos acima:

- Não podem ser apresentados como sendo "ideais" para a alimentação do lactente, seja através de ilustrações, fotos ou imagens de bebês;

- Não devem conter mensagens que possam por em dúvida a capacidade da mulher de amamentar seu filho,

- Devem conter frases definidas na Norma que enfatizam a superioridade da amamentação e subordinam o uso do produto à orientação de médico ou nutricionista.

De acordo com os especialistas em Nutrição, a maioria dos bebês não necessita de qualquer outro alimento além do leite materno durante os primeiros seis meses de vida. Por isso, nenhum alimento complementar deve ser promovido para o uso antes. dessa idade. Outros líquidos não são necessários, mesmo em climas quentes e secos (MARTINES et al., 1992). Estudo realizado em Pelotas, Rio Grande do Sul (VICTORA et al., 1987) mostra que a simples introdução de água ou chá reduz a proteção conferida pela amamentação.

Depois dos seis meses, quando então devem ser introduzidos outros alimentos, o leite materno ainda é o melhor líquido para os bebês. $\mathrm{O}$ uso de alimentos industrializados é cada vez mais comum, sendo obrigatório que a informação contida nos rótulos de alimentos infantis seja clara, acurada e na linguagem adequada, e que os rótulos não idealizem a alimentação artificial e o uso de mamadeira.

O Código Internacional da OMS/UNICEF alerta que os rótulos de todos os produtos que possam interferir com o aleitamento materno devem "ser elaborados com o objetivo de proporcionar a informação necessária sobre uso apropriado do produto e não desencorajar a amamentação" (ORGANIZAÇÃO..., 1981).

Imagens de bebês gordinhos e felizes foram utilizadas nas embalagens de leite e de outros produtos para alimentação infantil. A partir de 
1981, muitos fabricantes removeram estas imagens dos rótulos. Entretanto, muitas vezes elas foram substituídas por outras imagens que ainda idealizam o uso do produto, como por exemplo, o ursinho da Abbott-Ross e as figuras presentes em muitas marcas de mamadeiras e chupetas.

Companhias como a Gerber mantém a imagem de bebê como logomarca. Nesta pesquisa, entretanto, nota-se que empresas nacionais, como a Kuka e a Neopan, retiraram a figura de bebê de suas logomarcas, recentemente.

Com a valorização do leite humano e o crescente conhecimento sobre a fisiologia da lactação, os fabricantes começaram também a se aproveitar deste fato e a utilizar expressões que procuram dar destaque à amamentação, mostrando semelhança da fórmula infantil com o leite humano e do bico da mamadeira com o seio materno.

Apresentamos a seguir os artigos da Norma referentes à rotulagem ${ }^{3}$, os aspectos que foram violados, os produtos e nossos comentários:

\section{Art. 8 - Rótulos dos produtos dentro da abran- gência}

- Nenhuma figura ou foto de bebê, ou outras imagens que possam idealizar a alimentação artificial,

- Nenhuma frase que possa colocar a mãe em dúvida quanto à sua capacidade de amamentar.

VIOLAÇÃO ao Art. 8 - Contém fotos, figuras de bebês ou imagens relacionadas a bebês:

-O produto Isomil, da Abbott, contémo desenho do ursinho. Dos alimentos complementares, encontramos imagens em quatro produtos: Arrozina, Aveia Soberana, Baby Tea, e Papito. Entre os bicos, Chuca e Neopan apresentam imagens de criança. Das 24 chupetas analisadas, 13 infringiam este artigo da Norma, entre as quais: Neopan e Nuk. No caso das mamadeiras, mais de $50 \%$ das 26 encontradas no mercado brasileiro infringiam este artigo, entre as quais: Curity, Kuka, Lillo, Neopan. Todos os conjuntos e copinhos de treinamento encontrados apresentam imagens infantis em desacordo com a Norma Brasileira. Marcas como Neopan e Curity encontram-se em processo de mudança, podendo ser encontradas no mercado as embalagens com e sem figuras de bebês.

VIOLAÇÃO ao Art. 8 - Contém frases do tipo "quando não for possível" ou similares:

- A fórmula infantil Isomil, da Abbott, traz como aviso importante "O melhor alimento para o lactente é o leite materno, exceto quando existe condições nutricionais ou metabólicas especiais, casos em que Isomil poderia ser prescrito".

- A fórmula Pregestimil, da Mead Johnson, na ilustração de preparo da mamadeira usa a palavra amamentação como sinônimo de alimentação com mamadeira "Tampar a mamadeira e guardar até momento da amamentação" e "Na hora da amamentação adicionar o pó à água...".

- A fórmula Prosobee, da Mead Johnson, traz como nota importante: "O leite materno normalmente o melhor alimento para lactentes".

- O alimento complementar Dextrosol, da Refinações de Milho Brasil, diz ser ideal para adoçar mingaus, papas, leite, chás ou sucos para crianças e que "O leite materno é o alimento mais puro saudável para o seu bebê. Caso tenha que, sob orientação médica, substituir ou complementar a amamentação da criança, use Dextrosol para adoçar a mamadeira...”.

- O bico e a mamadeira Curity trazem a mensagem "Há casos, todavia, em que o médico recomenda que o aleitamento seja suplementado por alimentação alternativa. Somente então, a mãe deve recorrer à mamadeira para suprir as carências nutricionais da criança".

- O bico Nuk vented teat for thicker feeds, vendido na Drogaria Araújo de Belo Horizonte, traz um folheto anexo com orientações da Clínica Leite Meu. Uma das observações importantes diz " $A$ amamentação natural é o melhor para o seu bebê entretanto, isto nem sempre é possivvel, neste caso um bico Nuk é a alternativa ideal".

(3) Na Norma Brasileira, os artigos de 8 a 14 são os que tratam de rotulagem; aqui apenas não trataremos do Artigo 14 , que se refere a produtos não destinados a comercialização, mas para distribuição à profissionais de Saúde no lançamento, como amostra grátis para avaliação profissional. 
- A mamadeira Ortochuca diz "A Chuca sente-se na obrigação de informar que nada substitui o aleitamento natural no peito materno, por tudo que isso significa, principalmente pela passagem de fatores imunológicos, porém, em caso de impossibilidade natural, o aleitamento artificial é uma realidade".

VIOLAÇÃO ao Art. 8 - Contém frases que idealizam o uso do produto para lactente:

- O leite de cabra da Capril São Marcos refere "o leite de cabra é excelente alimento e por apresentar reação alcalina, é eficiente na prevenção de cólicas do recém-nascido".

- A aveia em flocos Soberana orienta o preparo de "Água de aveia para o bebê: ... para bebês a partir de 3 meses".

- O creme de arroz Colombo traz os dizeres " $e ́$ um produto alimentar indispensável à primeira infância; após o desmame ou conforme a indicação do médico use creme de arroz na alimentação infantil para o período de 1 a 4 meses; Lembre-se que a partir do $3^{\circ}$ ou $4^{\circ}$ mês as papinhas de legumes começam alternar-se com as mamadas".

- A chupeta Mini Mam orthodontic traz "Especialmente desenhada para atender às necessidades particulares dos recém-nascidos antes do sexto mês ou da chegada do primeiro dente".

\section{Art. 9 e 12 - Rótulos de fórmulas infantis e especiais}

Devem exibir com destaque as frases:

1. "O ALEITAMENTO MATERNO EVITA INFECÇÕES E ALERGIAS E FORTALECE O VÍNCULO MÃE E FILHO”

2. "ESTE PRODUTO SÓ DEVE SER UTILIZADO PARALACTENTESQUANDOORIENTADO PORMÉDICO OU NUTRICIONISTA"

Fica vedado usar os termos "humanizado", "maternizado", "substituto do leite materno" ou similares. produto.

Não indicar condições de saúde para uso do

As fórmulas infantis e os tipos de leite de seguimento normalmente são dados por mamadeira, precisam ser preparados com água (necessitando esterilização), são caros e podem ser diluídos, além de introduzirem o risco associado à retirada da proteção conferida pelo leite materno.

Quanto ao leite de seguimento, o desenho e o nome assemelham-se à fórmula padrão acrescido do número 2 (Nan 2, Nestogeno 2). A similaridade pode facilmente confundir o consumidor. Esta confusão pode levar alguns pais a utilizarem o leite de seguimento no lugar da fórmula convencional, particularmente se o preço estiver mais baixo.

Uma estratégia para ampliar a fatia de mercado é a diversificarão da mesma linha de produtos. No Brasil, a Nestlé tem produzido uma série de Nans e de Nestogenos, que a própria companhia chama de "família". O Codex Alimentarius, padrão para alimentos da OMS, declara que os leites de seguimento não devem ser usados antes da idade de seis meses.

\section{VIOLAÇÃO ao Art. 9 - Não contém as frases exigidas}

O produto Isomil, de Abbott Laboratórios do Brasil Ltdae Nursoy, de Laboratórios Wyeth-Whitehall Ltda.

VIOLAÇÃO ao Art. 9 - Contém a mensagem DESDE O NASCIMENTO, em destaque na face principal da lata, o que enfraquece a advertência exigida pelo artigo:

Os produtos Nan 1, Nan Ha, Nestogeno 1, e Pré Nan da Nestlé Industrial e Comercial Ltda. Além disso, o Nan 1 e o Nestogeno 1 têm os dizeres "é um leite infantil de início para lactentes sadios" e no Pré Nan "éum alimento dietético especialmente preparado para bebês muito pequenos e prematuros".

O leite de seguimento Nan 2 e Nestogeno 2, da Nestlé Industrial e Comercial Ltda, contém a mensagem "DESDE O $5^{\circ}$ MÊS" em destaque na face principal da lata. O leite de seguimento Nestogeno Soy contém a mensagem “A PARTIR DE 3 MESES”, em destaque na face principal da lata, além da frase "é um leite infantil de seqüência para bebês a partir de 3 meses, representando a parte líquida da dieta durante e após o desmame". Estes dizeres enfraquecem as mensagens exigidas pelo artigo 9, e 
desrespeitam as recomendações da Assembléia Mundial de Saúde:

"A prática de se administrar aos lactentes leites especialmente elaborados (chamados de "leites de seguimento"), que se está implantando em alguns países é desnecessária" (WORLD..., 1986).

\section{VIOLAÇÃO ao Art. 12 - Indicam condições de} saúde para sua utilização:

Os produtos Enfalac Prematuro, da BristolMyers Squibb Brasil S.A., Nursoy, de Laboratórios Wyeth-Whitehall Ltda e Pré Nan, da Nestlé Industrial e Comercial Ltda.

\section{Arf. 10 - Rótulos de leites não modificados}

Devem conter as seguintes mensagens:

- "Leite desnatado: ESTE PRODUTO NÃODEVE SER USADO COMO FONTE DE ALIMENTAÇÃO DO LACTENTE".

-"Leites semi-desnatados e padronizados: ESTE PRODUTONÃODEVE SER USADO COMO UNICAFONTE DE ALIMENTAÇÄO DO LACTENTE".

- "Leite integral: ESTEPRODUTONÀO DEVESER USADO COMO UNICA FONTE DE ALIMENTAÇÀO DO LACTENTE, SALVO SOB ORIENTAÇÃO DO MÉDICO OU NUTRICIO-NISTA".

VIOLAÇÃO ao Art. 10 - Não contém a mensagem exigida

O leite de cabra, da Capril São Marcos e Scabra leite integral de cabra, da Jackson Mitchell (EUA).

A maioria dos rótulos de leites não modificados contém as mensagens exigidas, porém com pouco destaque. Algumas vezes as mensagens têm pequenos erros,como a utilização da palavra "lactante" em vez de lactente (leite Conaprole da Cooperativa Nacional de Produtos de Leite, Montevidéu), ou "profissionais de saúde" no lugar de médico e nutricionista (leite Porto Fino da Nutrir Produtos Alimentícios Ltda).

\section{Art. 11 - Rótulos de alimentos complementares}

Devem exibir as frases de advertência:

1. "O ALEITAMENTO MATERNO DEVE SER MANTIDO APÓS A INTRODUÇÃO DE NOVOS ALIMENTOS NA DIETA DA CRIANÇA, ATÉ COMPLETAR DOIS ANOS DE IDADE OU MAIS".

2. "ESTE PRODUTO NÃO DEVE SER UTILIZADO NA ALIMENTAÇAO DE LACTENTES NOS PRIMEIROS SEIS MESES DE VIDA, SALVO SOB ORIENTAÇÃO DO MÉDICO OU NUTRICIONISTA".

\section{VIOLAÇÃO ao art. 11 - Não contém as mensagens exigidas:}

Os alimentos aveia em flocos finos da Quaker Brasil Ltda; Aveia em flocos Soberana de Produtos Alimentícios Corsetti S.A. Ind. e Com.; Aveia em flocos finos da Yoki Alimentos S.A.; baby tea do Laboratório Farmacêutico Flora da Índia Ltda; Creme de arroz da Arisco Produtos Alimentícios; Creme de arroz Colombo e Rozil da Confeitaria Colombo Dextrosol e Maizena Nutre (amostra) da Refinações de Milho Brasil; Farinha Láctea, Mucilon Arroz e Mucilon Milho da Nestlé Industrial e Comercial Ltda e Papito amido para mingaus da Macropack S.A.

A Nestlé lançou o Cerelac no Brasil há poucos anos. A partir de então, a estratégia foi comercializar o Cerelac para lactentes, redirecionando para outras faixas etárias produtos como a Farinha Láctea, o Mucilon Arroz, o Mucilon Milho e o Neston. As propagandas destes produtos não têm sido dirigidas a lactentes, entretanto, pensando na mãe consumidora, o mais adequado seria manter as mensagens exigidas pelo artigo, uma vez que durante muitos anos estes produtos foram comercializados para crianças pequenas. Quanto ao Cerelac, embora contenha as mensagens do artigo 11, tem em destaque a frase "DESDE O 6- MÊS" quando deveria ser "A PARTIR DO 6- MÊS".

É importante lembrar, no caso dos alimentos complementares, a decisão da Assembléia Mundial de Saúde de 1996:

"Os estados membros deverão...

assegurar que os alimentos com- 
plementares não sejam comercializados ou utilizados de maneira a prejudicarem a amamentação exclusiva e a continuidade da amamentação" (WHA 49/15).

\section{ARTIGO 13 - Mamadeiras, bicos e chupetas}

. Devem ter embalagens e/ou rótulos;

. Os rótulos não devem conter expressões que sugiram semelhança com o seio materno,

- Os rótulos devem exibir a mensagem "A CRIANÇA AMAMENTADA AO SEIO NÃO NECESSITA DE MAMADEIRA E DE BICO”.

Muitas chupetas podem ser encontradas à venda sem nenhuma embalagem ou rótulo; outras possuem embalagem inadequada e texto ilegível(bicos Ideatex, mamadeiras CIC).

Mamadeiras como a CIC orientam "Só amamente à noite se o médico achar conveniente". "CUIDADO muitos acidentes tem ocorrido porque a mãe dorme amamentando e sufoca a criança".

A Marwil Tortinha diz "a posição oblíqua do bico da Mamadeira Tortinha permite que a mamãe segure com uma posição mais cômoda, evitando cansaço enquanto o bebê se alimenta com a calma necessária".

Existem no mercado até mesmo mamadeiras que imitam garrafas de refrigerantes como a Munchkin Pepsi, alertando o consumidor" Nunca use a mamadeira com refrigerantes ou qualquer outro líquido sem valor nutritivo".

A mamadeira Ortochuca traz em destaque "Pediatras, ortodontistas e fonoaudiólogos reconhecem as qualidades da Ortochuca. Comprove!!!"

VIOLAÇÃO ao Art. 13 - Não contém a mensagem exigida:

- Bicos: Ideatex, da Ideatex Ind. e Com. Ltda; Kuka e Kuka Ortodôntico, da Kuka Produtos Infantis Ltda; Lillo miniform, da Hiborn do Brasil S.A., "Nuk vented teat for thicker feeds", da Drogaria Araújo e Prolar, da Comercial Utilicenter Ltda.
- Chupetas: Quatorze marcas, entre as quais: Argos, da Ind. Têxtil Belmar Ltda; Baby e Baby Orthodontic, da Start Baby Confecções Ltda e Baby 2000, Chicco, Nuk etc.

- Mamadeiras: Onze marcas, entre as quais: Argos, da Ind. Têxtil Belmar Ltda; Bambino, da Ind. e Com. Santa Thereza Ltda, Curity cristalina e Curity para água e chá, da CP Têxtil Ind. e Com. Ltda; Marwil, da Dermiwil Ind. Plástica Ltda, etc.

- Conjuntos e copo de treinamento: da Chuca Produtos Infantis Ltda, da Gerber (Best Prod. Exp. Imp. Ltda) e Copo Glug Glug, da Sociedade Industrial de Plásticos Ltda.

VIOLAÇÃO ao art. 13 - Contém expressões que sugerem semelhança com o seio materno:

- Bicos: Neopan látex, da Neopan Artigos Infantis Ltda e "Nuk vented teat for thicker feeds" da Drogaria Araújo de Belo Horizonte

- O bico "Nuk vented teat forthicker feeds" diz: "o formato cientificamente desenvolvido é baseado na forma do bico do seio da mulher quando amamenta".

- O bico Neopan látex: “Quase tão perfeito quanto o seio materno, ..."

- Mamadeiras: Lillo super mamadeira, da Hiborn do Brasil S.A. e Ortochuca, da Chuca Produtos Infantis Ltda.

- A supermamadeira Lillo: "Bico como desenho do seio materno: por isso o bebë não engole ar e mama como no seio."

- A mamadeira Ortochuca: "Sulco anatômico: assim como o seio materno é maleável, este sulco... Formato anatômico que estimula sucção igual a executada no seio materno". Além disto, traz um desenho comparando bebê mamando no peito e na mamadeira.

\section{CONCLUSÕES}

Em comparação a monitoramentos anteriores realizados em 1986/1987, 1989/19904 e 1993/1994, notamos que houve uma melhora na rotulagem dos produtos fabricados nacionalmente. De fato, até 1990, notava-se que a grande maioria das indústrias de

(4) Dados não publicados. 
mamadeiras, bicos e chupetas, assim como de alimentos infantis e de leites integrais e esterilizados não se haviam adequado às normas de rotulagem vigentes desde dezembro de 1988 (por exemplo, quanto a não inclusão de fotos ou imagens de bebês). Alegações de que as mensagens a serem colocadas não eram claras facilitavam esse fato.

Por outro lado, as produtoras multinacionais de fórmulas infantis, de maneira geral, sempre cumpriam alguns aspectos do Código Internacional e também da Norma Brasileira. Este fato se explica por todo o antecedente de luta de autoridades da Saúde e consumidores pela regulamentação do então considerado maior substituto do leite materno, a fórmula infantil. Em diferentes ocasiões, especialmente nas Assembléias Mundiais de Saúde, outros produtos utilizados como substitutos foram discutidos, para que fossem incluídos na abrangência do Código, e a Associação das Indústrias de Fórmulas Infantis (IFM) sempre foi contra.

É preocupante, atualmente, que mesmo com regras claras, e a exigência de que ao lançamento de um novo produto, o rótulo deve ser aprovado pela Vigilância Sanitária, tem havido rotulagem que contraria a Norma Brasileira. Observou-se, também, relançamentos ou re-embalagens de velhos produtos, em que certamente a não observância pode passar desapercebida.

Formas de idealizar ou mesmo mostrar uma aparente adequação à Norma para logo a seguir "enfraquecer" a mensagem favorável à amamentação têm sido freqüentes. Isto tem ocorrido com as fórmulas infantis, como é o caso da Abbott-Ross, que mantém a mesma estratégia no Peru, Paquistão, Malásia, África do Sul e Zimbábue (Afirma a superioridade do leite materno no rótulo, mas em seguida deixa a dúvida "para condições especiais...use...Enfamil") (INTERNATIONAL..., 1994).

Foram importantes também os achados quanto aos "leites de seguimento" (follow-on milks) que, por não existirem à época em que o Código foi aprovado, estão aparentemente fora da abrangência deste. Eles se dirigem a um mercado que a indústria tenta preservar para a criança mais velha, mas, na maioria das vezes, utilizando o mesmo nome da fórmula infantil, promovendo-a. A Companhia Nestlé, que detém de 35 a $50 \%$ do mercado de alimentos infantis no mundo, tem se destacado por essa prática no Brasil, e em outros países, como no Paquistão(Nan 1 e 2), Filipinas, Bolívia e Gana (com o Nestogeno 1 e 2) (INTERNATIONAL..., 1994).

$\mathrm{Na}$ mesma linha foram os achados referentes às fórmulas para necessidades especiais, como é o caso dos leites para prematuros, onde a Companhia usa o mesmo nome com a adjetivação pré. Isto ocorre também na Indonésia e Índia.

A questão da advertência nos rótulos do leite integral, semi-desnatados, ou outros fluidos similares, é certamente um problema a ser revisto pelas autoridades sanitárias, porque não existe regulamentação quanto ao tamanho ou proporcionalidade das letras dessa advertência. Conforme registramos, a indústria tem colocado dizeres freqüentemente ilegíveis, o que é inaceitável do ponto de vista da Vigilância Sanitária. Esta questão é comparável às advertências contidas nos maços de cigarro que, antes da regulamentação, traziam frases de advertência na cor do fundo do pacote, tornando-a quase invisível; isto precisou ser regulamentado para que houvesse contraste e destaque.

No caso de mamadeiras, chupetas e bicos, a não obediência a normas originadas no Ministério da Saúde tem sido a regra mesmo em outros países. Procedimento análogo foi relatado com relação à Curity, na África do Sul, ou a Evenflo, no México. A Nuk, na África do Sul, também compara seu "bico" ao seio materno, como faz aqui. Hoje está claro o maior risco de desmame (de 3.84 vezes) com um mês de vida entre usuários de chupeta comparado a crianças que não a usam (BARROS, 1995). Por essa razão, esforços das autoridades internacionais de saúde ficaram bastante definidas nos "10 passos para o sucesso do Aleitamento Materno", onde o passo $9^{\circ}$ diz: NÃO DAR BICOS OU CHUPETAS A CRIANÇAS AMAMENTADAS AOSEIO(ORGANIZAÇÃO..., 1989).

Os resultados deste monitoramento proporcionam subsídios à atuação mais efetiva dos órgãos fiscalizadores do governo: a Secretaria Nacional de Vigilância Sanitária do Ministério da Saúde, Ministério da Agricultura e Delegacias de Defesa do Consumidor. As violações aqui analisadas contribuem também para o trabalho de voluntários, como os da International Baby Food Action Network (IBFAN), rede que atua em mais de 70 países na defesa do consumidor infantil, pelo cumprimento do Código Internacional da OMS/LINICEF. A repetição periódica 
de monitoramentos como este é importante para a contínua vigilância de regras sanitárias essenciais, desta forma buscando garantir que os substitutos da amamentação e do leite materno sejam comercializados dentro de princípios éticos.

\section{AGRADECIMENTOS}

Às estagiárias Cintia Gisela Bezuti e Daniela Wenzel, alunas de Nutrição, Faculdade São Camilo.

\section{REFERÊNCIASBIBLIOGRÁFICAS}

BARROS, F.C. et al. Use of pacifiers is associated with decreased breastfeeding duration. Pediatrics, Evanston, v.95, n.4, p.497-499, 1995.

CHETLEY, A. The politics of baby, foods: successfull challenges to an international marketing strategy. London: Frances-Pinter, 1986.189p.

DALY, F.H. Condensed milk. Lancet, London, v.2, p.653, 1872.

DOBBING, J. (Ed.) Infant feeding: anatomy of a controversy 1973-1984. Berlin: Springer-Verlag,1988. $169 \mathrm{p}$.

GOLDENBERG, P. Repensando a desnutrição como questão.social.Campinas:UNICAMP,1988.159p.

GREINER,T. The promotion of bottle feeding by multinational corporations. Ithaca: Cornell University, 1975.82p. (Cornell International Nutrition Monography, series n.2).

INTERNATIONAL BABY ACTION NEWTWORK. Breaking the rules. Cambridge UK, 1994. 42p. (Baby Milk Action).

INTERNATIONAL CODE DOCUMENTATION CENTRE. ICDC Update. Penang, Malásia,1996. 4p. (July).
INSTITUTONACIONALDEALIMENTAÇÃOENUTRIÇÃO. Norma Brasileira para Comercialização de Alimentos para Lactentes. 2.ed. Brasília: Ministério da Saúde,1993.20p.

JELLIFFE, D.B. Commerciogenic malnutrition. Nutrition Review, NewYork, v.30,n.9, p.199-205, 1972.

KUCINSKI, B., LEDOGAR, R.J. Fome de lucros. São Paulo: Brasiliense, $1977.254 \mathrm{p}$.

MARTINES, J.C. et al. Breast feeding in the first six months: no need for extra fluids. British Medical Jornal, v.304, p.1068-1069, April 1992.

ORGANIZAÇÃOMUNDIALDESAÚDE/LIMCEF.Código internacional de comercialização de substitutos do leite materno. Genebra,1981.38p.

Proteção, promoção e apoio ao aleitamento materno: o papel especial dos serviços materno-infantis. Genebra, 1989.32p.

PALMER, G. The politics of breastfeeding. London:Pandora Press, 1988.304p.

PLATT, W.H.Condensed milk and artificial feeding. Lancet, London, v.2,p.695, 1872.

REA, M.F. Substitutos do leite materno: passado e presente. Revista de Saúde Pública, São Paulo, v.24, n.3, p.241-249,1990.

TOMA, T.S. (Coord.) Violando a norma 1996: relatório nacional das violações à Norma Brasileira para Comercialização de Alimentos para Lactentes. São Paulo:IBFAN/UNICEF, 1996.38p.

VICTORA, C.G. et al. Evidence for protection by breastfeeding against infant deaths from infectious diseases in Brazil. Lancet, London, p.319-322, Aug. 1987.

WORLD HEALTH ORGANIZATION. Resolution WHA 39,28,16 May 1996. Geneva,1996.(39/vr/15).

Recebido para publicação em 1 de outubro de 1996 e aceito em 24 de abril de 1997. 